\title{
Automatic Environmental Monitoring System using Wireless Sensor Network
}

\author{
Soniya Sunny \\ Department of Electronics and Communication Engineering \\ Believers Church Caarmel Engineering College, Pathanamthitta, Kerala, India \\ Rejin Mathew \\ Department of Electronics and Communication Engineering \\ Believers Church Caarmel Engineering College, Pathanamthitta, Kerala, India \\ Kuruvilla John \\ Department of Electronics and Communication Engineering \\ Believers Church Caarmel Engineering College, Pathanamthitta, Kerala, India
}

\begin{abstract}
Wireless sensor networks provide many solutions for various real time applications. In this paper, a real time environmental monitoring application is presented. Sensors are used to collect details regarding environmental conditions like temperature, humidity, pressure etc. at different locations. The real time data is given to Raspberry Pi which acts as a control station. It stores the data collected from different sensor units and analyzes the stored data. ZigBee protocol is used for communication between different sensor nodes. The system also provides a web interface for the user so that the user can control and monitor the system remotely.
\end{abstract}

Keywords - Environmental Monitoring, Wireless Sensor Network, Raspberry Pi

\section{INTRODUCTION}

The function of a sensor is to convert a physical quantity into digital data. When different types of sensors are integrated to a particular device to collect data from a location, we call it as a sensor node. To collect data from large indoor or outdoor environment, many sensor nodes can be organized into a distributed network, which can be referred as a sensor network. There must be a coordinator node to monitor and control data from all sensor nodes. There must be an active communication system for transferring data regarding different locations. In this paper, an application meant for environmental monitoring is presented. Environmental conditions like temperature, pressure, humidity etc. can be monitored using wireless sensor networks from remote locations.

Wired communication is not possible for the sensor nodes that are residing far from the user. Recently, many wireless technologies are evolved, e.g. Wi-Fi, ZigBee, Bluetooth etc. the wireless devices that are using such technologies have different range, data rate, power consumption and other features. Though they are small and can be easily connected to a sensor node.

Literature review is explained in section II. System architecture is presented in section III. Design of control station and end device sensor node is then presented. System operation is explained in section VI. Concluding remarks are given in section VII.

\section{LITERATURE REVIEW}

From the literature, we can see the application of Wireless Sensor Network (WSN) technology for large scale and long duration environmental monitoring [1]. Environmental monitoring system should fulfill some technical requirements like high level of performance, system integration, reliability, accuracy, productivity, flexibility, robustness etc. different designs of sensor networks for environmental and habitat monitoring are discussed in the literature [2]. The embedded construction and distributed nature of wireless sensor networks find its advantages over traditional sensing techniques. It can also employ mesh networking scheme and can offer better coverage than other centralized sensing technologies. Some WSNs uses ZigBee protocol for communication between different sensor nodes [3]. Raspberry Pi can be used as the control section which interconnects several sensor nodes and enables 
effective communication between them [4]. Raspberry Pi that works on open source Linux operating platforms can be used as a base station which connects the wireless sensor networks and other networks.

\section{SYSTEM ARCHITECTURE}

Wireless senor network system involves development and integration of many hardware and software components. Figure 1 shows the overall system architecture of WSN system for environmental monitoring [5]. This system consists of raspberry pi, many distributed wireless sensor nodes, ZigBee protocol etc. Raspberry pi that acts as a base station interconnects multiple sensor nodes. Each sensor node is combination of sensors, microcontroller and zigbee transceiver module (Xbee). Sensor node collects and distributes information or sensor data. In addition, there is user application program on each sensor node which handles data from sensors in a well-defined manner and communication with base station. Raspberry Pi interacts with multiple sensor nodes. The client can interact with the system through Raspberry Pi.

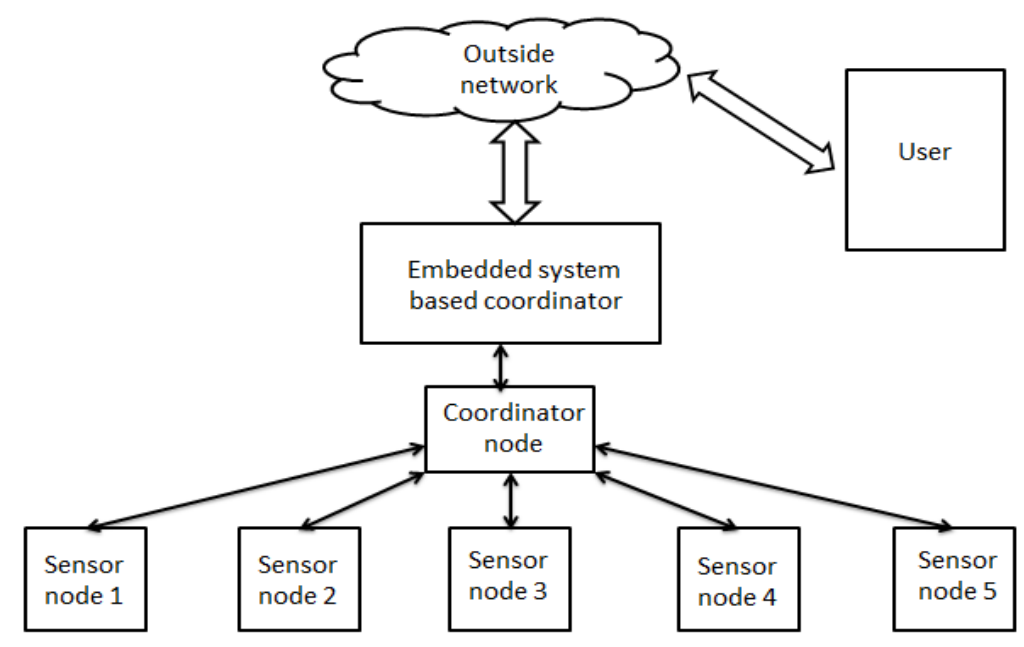

Figure1. System architecture

\section{A. Raspberry Pi}

Raspberry Pi is the cheapest ARM11 powered single board computer which uses Linux operating system. It runs an ARM11 microcontroller at $700 \mathrm{MHz}$ and has a 512 Mega Bytes of RAM memory [6] [7]. The Raspberry Pi is a low cost, low power credit card sized single board computer [8] [9]. In this project, Raspberry Pi B+ model [9] (fig. 2 ) is used as this model has better specifications as compared with other Raspberry Pi models. It supports a number of operating systems including a Debian-based Linux distro, Raspbian which is recommended by raspberry pi foundation, which is used in our design. Raspberry Pi can be connected to a local area network through USB Wi-Fi adapter or Ethernet cable, and then it can be accessed by more than one user from anywhere in the world through SSH remote login or by using putty software by just putting raspberry pi IP address on it. The raspberry pi is booted by external SD card or micro SD card [8] [9]. 


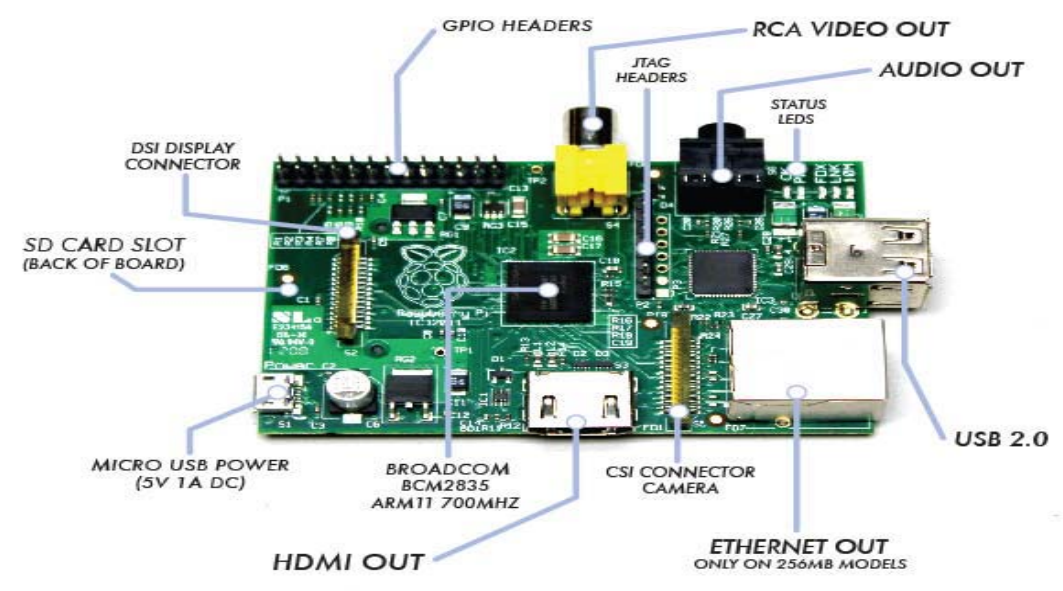

Figure 2. Raspberry Pi

\section{B. Sensor node}

Sensor node is used in the end device side to sense the environmental parameters. It is designed using PIC16F877A microcontroller. It also consists of ZigBee protocol based radio transceiver unit, temperature sensor, humidity sensor, pressure sensor and power supply unit. It will sense parameters at regular intervals and will send the data to the coordinator node through ZigBee wireless communication protocol [10].

\section{Zigbee}

ZigBee is a specification for a suite of high-level communication protocols used to build wireless networks from small, low-power digital radios. It is a wireless protocol based on an IEEE 802.15 .4 standard. It is a simple, efficient, reliable, low cost and low power standard of wireless technology. Though its low power consumption limits transmission distances to 10-100 meters line-of-sight, based on environmental characteristics and power output, ZigBee devices can transmit data signals over long distances by passing data through a mesh network of intermediate devices to reach more distant ones if needed. ZigBee has a defined rate of $250 \mathrm{Kbit} / \mathrm{s}$, best suited for intermittent data transmissions from a sensor device or input device [11]. The technology defined by the ZigBee specification is supposed to be simpler and less expensive than other wireless personal area networks (WPANs), such as Wi-Fi or Bluetooth. For example DigiXbee series modules S1 and S2 implement the IEEE 802.15.4 radio and ZigBee networking protocol [12]. Here we used XBee series module S2 from Digi international which fully implement Zigbee protocol. XBee series S2 module also covers more area than XBee S1 module.

\section{Sensors}

Different types of sensors are placed at each sensor nodes to monitor the environmental conditions at different locations. LM35 is used as the temperature sensor. The LM35 outputs an analog voltage proportional to the temperature values. The output from the LM35 is $0.1 \mathrm{~V} /{ }^{\circ} \mathrm{C}$. So if the temperature sensed is $61{ }^{\circ} \mathrm{C}$, the output voltage is $0.61 \mathrm{~V}$. This analog voltage is read by the PIC microcontroller. SYH-1 is the humidity sensor used. It has an operating humidity range of $20-90^{\circ} \mathrm{C}$. Storage humidity range of SYH-1 is less than $95^{\circ} \mathrm{C}$. it has a maximum $0.26 \mathrm{~mW}$ rated power.

\section{E. Data base and web server}

In the proposed system, we have designed the database based on MySQL which is installed on Raspberry Pi. MySQL is the popular choice of database in a web application. MySQL is a relational database management system (RDBMS) which is an open source software. Database stores the environmental parameter information send by a sensor node to it continuously. It provides the information to the web page for monitoring the system remotely [10]. 


\section{Design of Control Station:}

Raspberry Pi acts as a base station which connects different sensor nodes by ZigBee communication protocol and user by internet. For wireless communication and multi-hop networking protocol, we used XBee series module from Digi international. XBee module is configured as a coordinator on the Raspberry Pi. Raspberry Pi can be connected to XBee module directly through USB cable and also by UART serial communication interface.

As XBee module can be configured into three types of devices: coordinator, router, and end device. Coordinator has the capability to control the entire network. The base station or control station also acts as a gateway in this application. The data collected or detected by sensor node sends to the control station and inserts the data received from sensor nodes into MySQL database of Raspberry Pi. Multiple users can access the Raspberry Pi through Ethernet or Wi-Fi connection within local area network or from anywhere on the internet. Figure 3 shows the functional block diagram of control station.

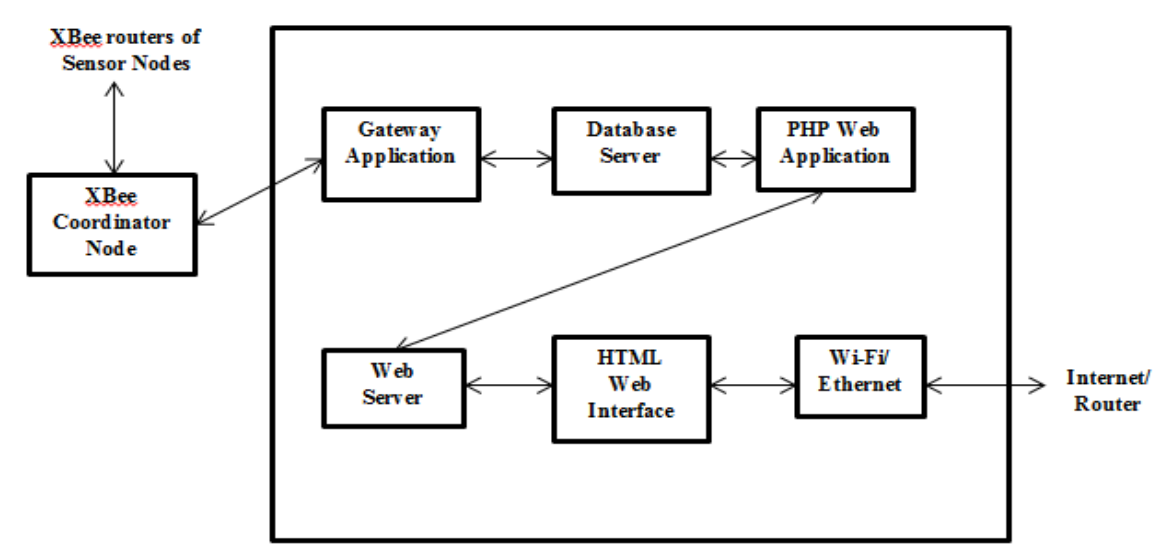

Figure 3. Functional block diagram of control station

\section{Design of End Device Sensor Node:}

PIC16F877A is used as the microcontroller in sensor node unit. The functional block diagram of sensor node is shown in figure 4. ZigBee devices are mainly suitable for fast prototyping for wireless sensor network applications. It is possible to build complex mesh network using these devices. We have to develop application both at base station and sensor nodes to use some advanced features of $\mathrm{ZigBee}$. We can connect number of sensors to the sensor node. The XBee module S2 is connected to sensor node board as shown in figure 5. The XBee module encapsulates 802.15.4 RF transceivers and ZigBee protocol stacks, and it can be easily integrated into any microprocessor or microcontroller systems such as Raspberry Pi through UART serial communication interface [12]. 


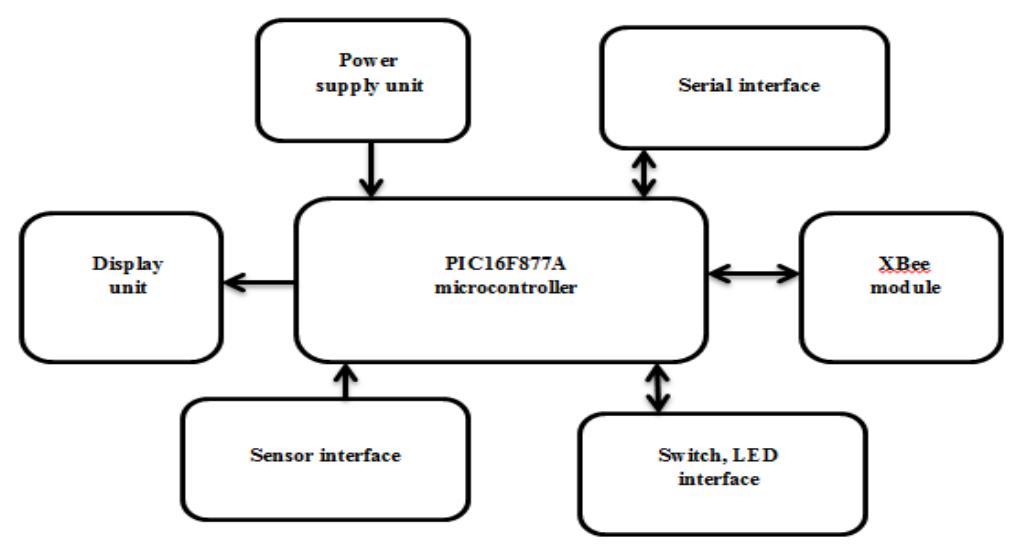

Figure 4. Functional block diagram of sensor node

The connection between controller of sensor node and XBee is shown in figure 5. The XBee module is configured as a router on the sensor nodes. Router can relay messages in a tree or mesh network and Coordinator has the capability to control the entire network. The XBee module can be configured into three types of devices: coordinator, router, and end device. Coordinator has the capability to control and initiates the entire network. Router can relay messages in a tree or mesh network topologies [4]. End device can only communicate with the coordinator or the router. There can be only one coordinator node in a network.

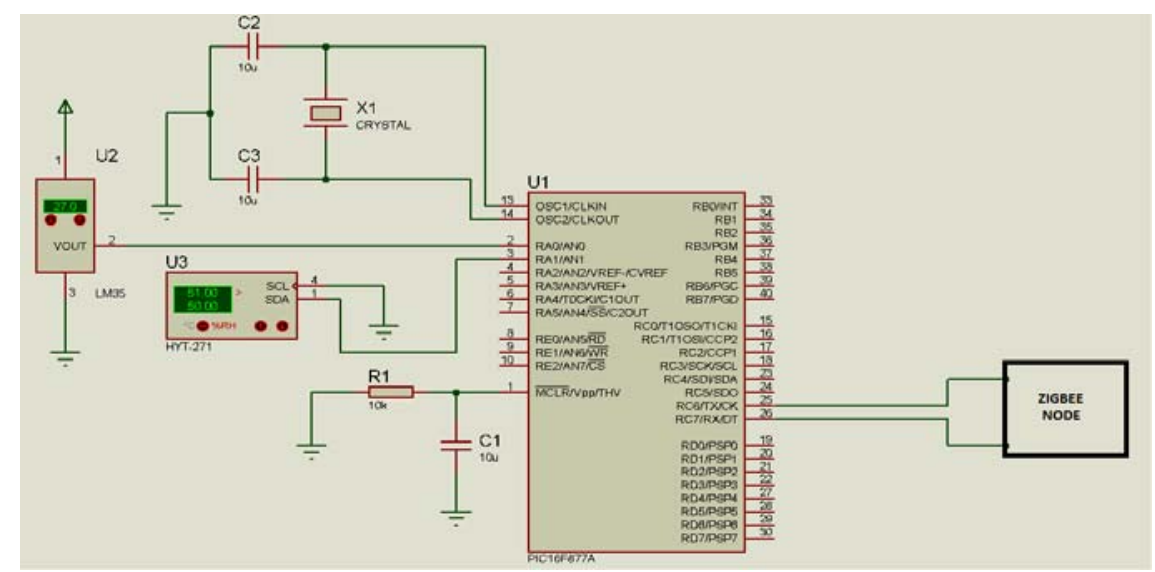

Figure 5. Connection between controller of sensor node and ZigBee node

\section{SYSTEM OPERATION:}

In WSN, there are three types of devices: coordinator, router and end tags. There is only one coordinator in the network, which actually communicates with the base station shown in figure 6 and there can be more than one router and end devices. So, here one Xbee is configured as a coordinator, which is connected with the Raspberry Pi using UART protocol. For simple experimental purpose, just two sensor nodes are configured as P1 and P2. One sensor node is configured as an end tag E52 in which light sensor is connected (can be connected any sensor) shown in figure 7 which will send its real time data to the nearest router. The router has the capability of routing, which means router sends its data to its nearest router. So, Raspberry Pi receives the sensor's data from its nearest router and also coordinator sensor node will show that from which end device the data comes. The data comes in the base station is stored into the table created in database of Raspberry Pi [4]. This table shown can be fetched from web browser/PHPMyAdmin by clients by using Raspberry Pi IP address. This table can be also seen in MySQL terminal of Raspberry Pi. 


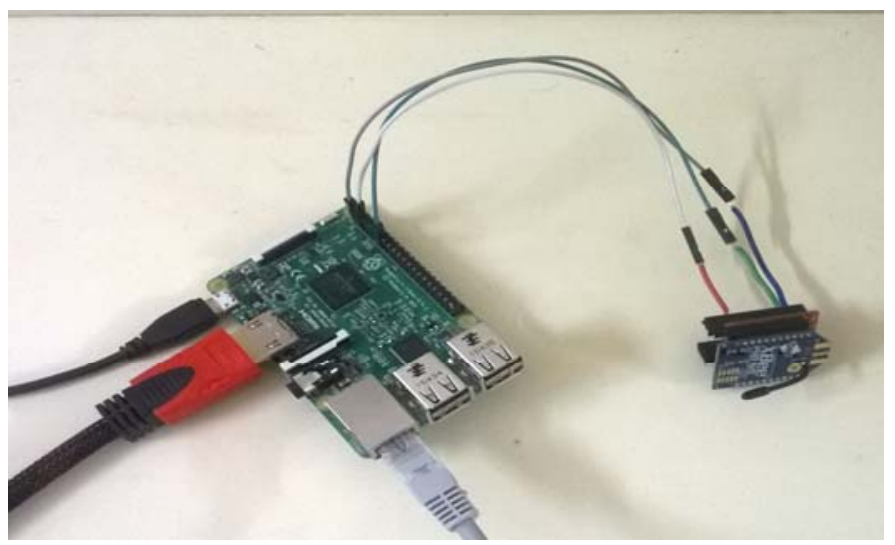

Figure 6. Base station

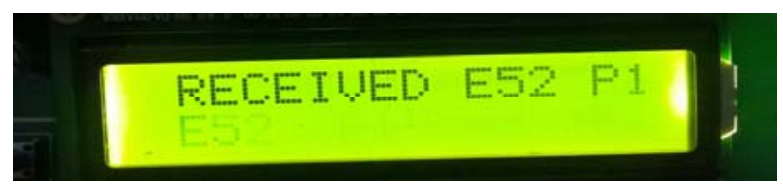

Figure 7. Router P1 received data sent by end tag E52

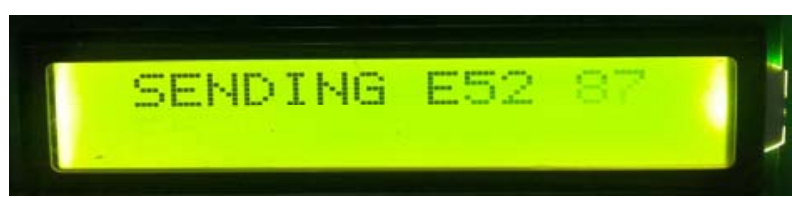

Figure 8. End tag E52 sending to nearest router

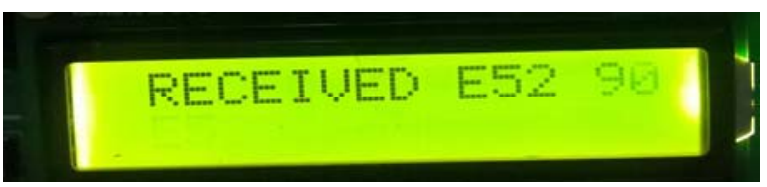

Figure 9. Router P1 received data sent by end tag E52

\section{CONCLUSION}

An automated environmental monitoring system is designed using wireless sensor networks. The design involves Raspberry Pi as an embedded Linux board which collects the data from different sensor nodes at regular intervals. This data is stored in the database and web interface is also provided to the user. Since web interface is provided, the client can easily monitor the system at any time and thus it helps to reduce human interventions. The sensor nodes communicate with the coordinator node through wireless ZigBee protocol. The system has low power consumption with better processing ability and high data transmission speed.

\section{REFERENCES}

[1] Prachi Sharma, "Wireless Sensor Networks for Environmental Monitoring", International Journal of Scientific Research Engineering \& Technology, IEERET-2014 Conference Proceeding, 3-4 November, 2014.

[2] Christos G. Panayiotou, Despo Fatta, Michalis P. Michaelides, "Environmental Monitoring Using Wireless Sensor Networks", InternationalJournal of Scientific Research Engineering \& Technology, November 2014

[3] Mr. Sudhir G. Nikhade, Dr. Mrs. A. A. Agashe, "Wireless Sensor Network Communication Terminal Based on Embedded Linux and Xbee", International Conference on Circuit, Power and Computing Technologies, 2014.

[4] Sudhir G. Nikhade, "Wireless Sensor Network System using Raspberry Pi and Zigbee for Environmental Monitoring Applications", International Conference on Smart Technologies and Management for Computing, Communication, Controls, Energy and Materials, May 2015. pp.376-381.

[5] Sheikh Ferdoush, Xinrong Li "Wireless Sensor Network System Design using Raspberry Pi and Arduino for Environmental Monitoring Applications", Elsevier The 9th International Conference on Future Networks and Communications (FNC-2014). 
[6] Vujovic, V.; Maksimovic, M., "Raspberry Pi as a Wireless Sensor node: Performances and constraints," Information and Communication Technology, Electronics and Microelectronics (MIPRO), 2014 37th International Convention on, vol., no., pp.1013,1018, 26-30 May 2014.

[7] Kochlan, M.; Hodon, M.; Cechovic, L.; Kapitulik, J.; Jurecka, M., "WSN for traffic monitoring using Raspberry Pi board," Computer Science and Information Systems (FedCSIS), 2014 Federated Conference on, vol., no., pp.1023,1026, 7-10 Sept. 2014.

[8] Powers, Shawn. "The open-source classroom: your first bite of raspberry pi." Linux Journal 2012.224 (2012):7.http://www.raspberrypi.org.

[9] RaspberryPi,webpage:http://en.wikipedia.org/wiki/Raspberry_Pi [April20, 2014].

[10] Pandurang H. Tarange, Rajan G. Mevekari, "Web based Automatic Irrigation System using wireless sensor network and Embedded Linux board", International Conference on Circuit, Power and Computing Technologies, 2015

[11] ZigBee Specification.ZigBee Alliance 2006.http:// www.zigbee.org/.

[12] Digilnternational Inc., available at http://www.digi.com. 